
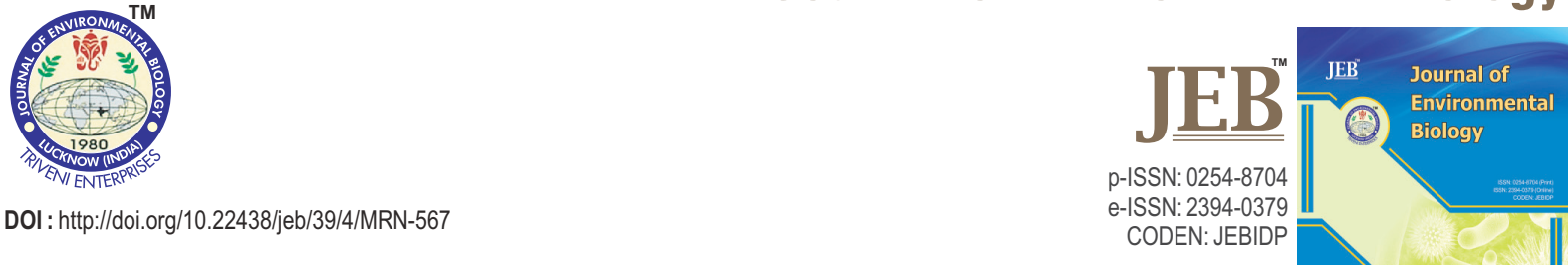

\title{
Study of soil nutrients, physical and biological characteristics of paddy fields after lifting measures: A case study of China's Ganjiang region
}
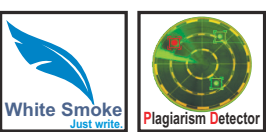

Authors Info

J. Zhang ${ }^{1 *}$, L. Han ${ }^{1}$, Y. Ji',
M. Duan', G. Cai ${ }^{2}$, J. Wu',
W. Zhu', G. Gao ${ }^{2}$, Y. Peng and Y. Zhou

${ }^{1}$ College of Environment, Hohai University, Nanjing-210 098, China

${ }^{2}$ College of Water Conservancy and Ecological Engineering, Nanchang Institute of Technology, Nanchang-330 099, China

${ }^{3}$ JiangXi Engineering

Research Center of Water Engineering Safety and Resources Efficient Utilization, Nanchang-330 099, China

${ }^{4}$ Shanghai Academy of Environmental Sciences, Shanghai-200 233, China

*Corresponding Author Email : jiyong@nit.edu.cn

Key words

Ganjiang region

Integrated fertility index

Lifting measure

Paddy soil

Soil nutrients

Publication Info

Paper received : 16.01.2017

Revised received : 26.06.2017

Re-revised received :06.09.2017

Accepted:07.11.2017

\section{Abstract}

Aim : Lifting measures combining stripping of soil surface with lifting of soil by stone and clay have been widely employed to solve land-flooding conflicts in water project construction. The aim of the present research was to demonstrate the physical, biological and nutrient characteristics of soil at different intervals after taking the lifting measures.

Methodology : Considering farm field cultivation, thirty nine sites of three different kinds were selected to reflect the effect of construction of the Xiajiang Water Control Project on soil characteristics. Soils were collected to test the physical, chemical, and nutrient properties. Soil quality indices were combined with statistical analyses to evaluate the effect of the lifting measures.

Results : After the lifting measures were employed, the quality of soil physical properties and nutrients, such as the level of soil moisture content, soil respiration intensity, soil organic carbon value and total nitrogen decreased significantly in the first year. Compared with nonlifted areas, both physical and chemical properties decreased to a certain degree. However, soil quality gradually recovered via continuous management and cultivation, and no significant difference between the lifted and non-lifted areas was observed after three years of cultivation based on the Integrated Fertility Index.

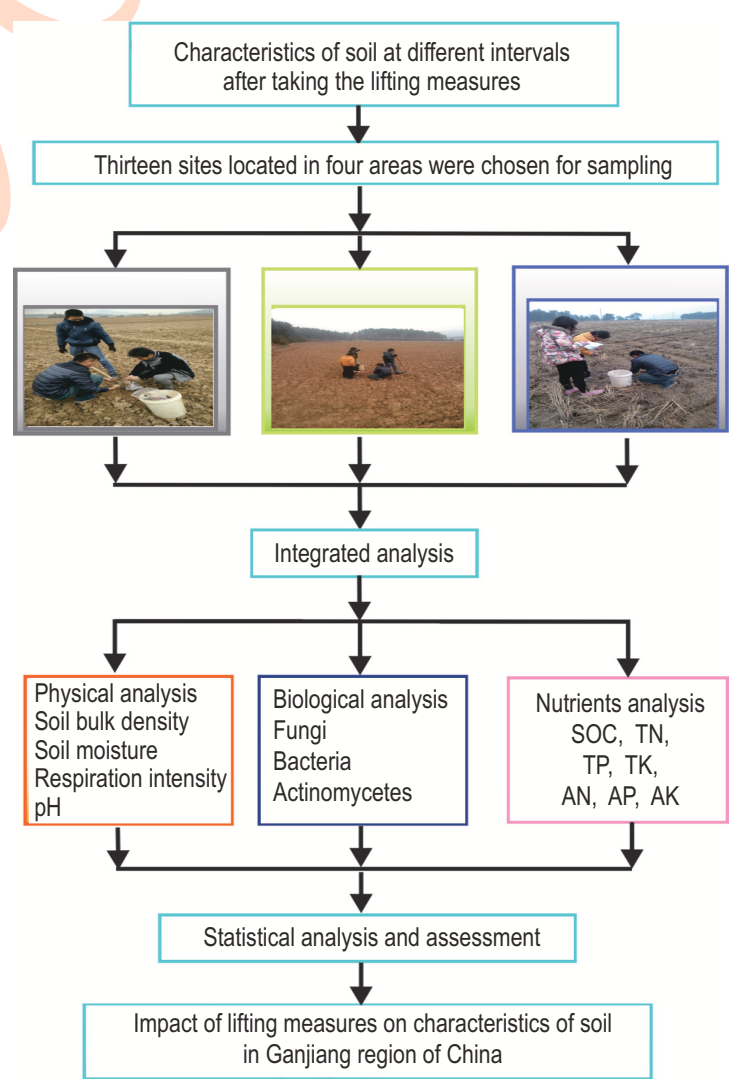

Interpretation : Lifting measures

can destroy the soil structure in the early stage and thus degrade the soil quality and nutrient cycles. Through scientific management and cultivation, soil physical and chemical properties could be gradually 


\section{Introduction}

Soil fertility is subject to the biological environment and presents individual differences in different places (Mandal et al., 2015). Nutrients and microorganisms in soil play an important role in improving soil quality (Sun et al., 2011). In the past several decades, the function of organic and microbial matter in soil has attracted extensive attention because of the positive effects on agricultural productiveness (Castilho et al., 2016). Biological properties of soil are closely related to biochemical properties that greatly contributed to decomposition processes (Hobara et al., 2014). Some studies have indicated that microorganisms can increase amino acid levels, whereas soil fungi can change the amount of macro aggregates and the structure of soil (Helfricha et al., 2015). Microorganisms are necessary for recycling within terrestrial ecosystems. In addition, soil organic matter (SOM) has been considered a complex compound of plant litters and microbial outputs in various stages of degradation (Masoom et al., 2016). It can be very effective in maintaining moisture in the soil, preventing soil salinization, and providing a medium environment for microorganisms (Mlih etal., 2016).

Many studies have revealed that rotational tillage can enhance the vertical distribution of total nitrogen (TN) and SOM stocks (Sutton and Sposito, 2006). Compared with conventional methods, rotational tillage decreases soil bulk density and positively influences biomass and productiveness (Hou et al., 2012). Some studies have also shown that effective management alleviates soil water deficit, increases crop productivity and improves soil physical properties (Lozano et al., 2016). Lifting measures are comparable to rotational tillage, both destroy the soil's original structure and greatly effect the soil quality. Since the 1970s, lifting measures have been used extensively in basins destroyed by Water Control Projects in China to resolve immigration and relocation issues at lower cost (Zhen, 2015). However, lifting measure application is usually limited to a small scale and does not cause obvious effects on the local environment. Nevertheless, few studies have been conducted to examine the effect on agricultural production caused by lifting.

The Xiajiang Water Control Project is the largest water conservancy program of the Ganjiang River, and aims in controlling flood, generating power and utilizing water resources for agricultural purposes. Its implementation can significantly enhance flood control capacity of cities located downstream of the project (Tang and Wang, 2003). However, this project faces a big challenge in terms of large areas of cultivable land that become submerged. Presently, these arable land areas are very important in guaranteeing food production for local residents. After wide discussion, lifting measures have been accepted as an alternative method for reducing the amount of submerged areas of arable lands. The objective of this study was to analyse the physical, chemical and biological properties of lifted soil, correlation between nutrients and properties, and soil quality at different stages using Integrated Fertility Index (IFI).

\section{Materials and Methods}

Study area : During the construction of Xiajiang Water Control Project, more than 1910.06 ha of farmland have been occupied. Among those occupied, approximatly 142.63 ha are reverting to forests and 1092.93 ha are undergoing lifting measure. Lifting measures involved three steps. First, the surface soil of original fields is stripped, collected and stored. Then, the original fields were lifted by adding stone layers and clay layers whose thickness was determined by hydrologic calculation. After compaction, the original surface soil was added back as a top layer for agricultural purposes. Based on the scientific field investigation, the surface arable layer was about $30 \mathrm{~cm}$ deep. Based on the recultivation period after lifting measures were applied, 13 sites located in four areas on the east side of the Reservoir Xiajiang, namely Shui Tian, Cha Tan, Liao Qiao, and Ge Shan, were selected for sampling. Of these sampling sites, 3 were in Shui Tian, 4 in Cha Tan, 4 in Liao Qiao and 2 in Ge Shan, respectvely.

Sample collection and preparation : Thirty nine samples were collected at the end of December 2014 from the four lifted areas using a Hydro-Bios stainless steel grab sampler and then wrapped in polyethylene plastic bags (Fig. 1). At each site, three samples, using a five-point sampling method with a 'W'-shaped distribution, were collected from the surface soil (0 to $20 \mathrm{~cm})$. The soil samples were designated $3 Y$ ( 3 years post-lifting), $1 Y$ (1-year post-lifting), $O Y(0$ years post-lifting), and BK (original field as blank) based on the cultivation period after lifting measures were applied. A portable Magellan GPS (Triton 300E) was used to record the global position information.

Sample analysis and processing : After gathering, the surface soil samples were well marked, immediately sent to the laboratory, and then air-dried. Stones, gravel, animal residues, plant roots and sticks were picked out. These samples were then ground with a mortar, passed through a $0.15 \mathrm{~mm}$ mesh sieve, and stored in a ziplock bag at $-4^{\circ} \mathrm{C}$ prior to laboratory analyses.

As mentioned in Masciandaro et al. (2013), the potassium dichromate external heating method, Kjeldahl method, molybdenum antimony colorimetric assay, flame photometry, alkaline hydrolysis diffusion method, flame photometry and sodium bicarbonate method were used to determine the levels of soil organic carbon (SOC), TN, total phosphorus (TP), total potassium (TK), alkaline hydrolysis nitrogen (AN), available potassium (AK) and available phosphorus (AP), respectively. After $20 \mathrm{~min}$ of sterilization, beef extract-peptone medium, improved Gause's No. 1 medium, and Martin agar medium were used to culture bacteria, actinomycetes and fungi, respectively. The improved Gause's No. 1 medium and Martin agar medium, $3 \%$ potassium dichromate (One $\mathrm{ml}$ per $300 \mathrm{ml}$ ) and $1 \%$ streptomycin (3.3 per $\mathrm{ml} 1000 \mathrm{ml}$ ) were added to inhibit the growth of bacteria and actinomycetes. Diluted samples were inoculated under sterile conditions and then moved into a constant temperature incubator. The cell counts for bacteria and 
actinomycetes were calculated after $10 \mathrm{~d}$ culture at $25^{\circ} \mathrm{C}$ and for the fungus after $5 \mathrm{~d}$ culture at $25^{\circ} \mathrm{C}$.

\section{Results and Discussion}

Appropriate physical and biological properties have positive effects on nutrient cycling and water transportation, transfer and storage. Several studies have revealed that the physical and biological properties of soil are closely related to the soil fertility level (Smebye et al., 2015). As shown in Fig. 2, the soil bulk densities of four soil types ranged from 1.09 to 1.87 $\mathrm{g} \cdot \mathrm{cm}^{-3}$ and their average values were in the following order: $0 \mathrm{Y}>$ $1 Y>3 Y>B K$. The soil moisture contents ranged from $6.96 \%$ to $31.27 \%$, and their average values ranked as follows: BK $>3 Y>1 Y>0 Y$. The trend for soil respiration intensity was same as that for soil moisture: $\mathrm{BK}>3 \mathrm{Y}>1 \mathrm{Y}>0 \mathrm{Y}$. The $\mathrm{pH}$ varied from 6.91 to 4.61 , with a mean value of 5.49. The $\mathrm{pH}$ values of $\mathrm{OY}$ areas were higher compared with those of $\mathrm{BK}$ and $3 \mathrm{Y}$ areas.

It is well acknowledged that microorganisms play an important role during the recycling process of soil nutrients. The quantity of organisms is determined by type of soil, soil layer thickness, type of vegetation, geographical climate and other factors. Therefore, the number of microorganisms could directly reflect the status of soil fertility (Mahanta et al., 2014). In soil, bacteria had the highest abundance, followed by actinomycetes and fungi. When compared with BK, no significant differences were found among the abundances of all the selected microorganisms of $1 Y$ and $3 Y$ (Fig. 3); which indicate that soil quality in $1 Y$ and $3 Y$ gradually increased after several years of cultivation and management. However, the abundance of fungi and bacteria in OY were still lower than those in BK.

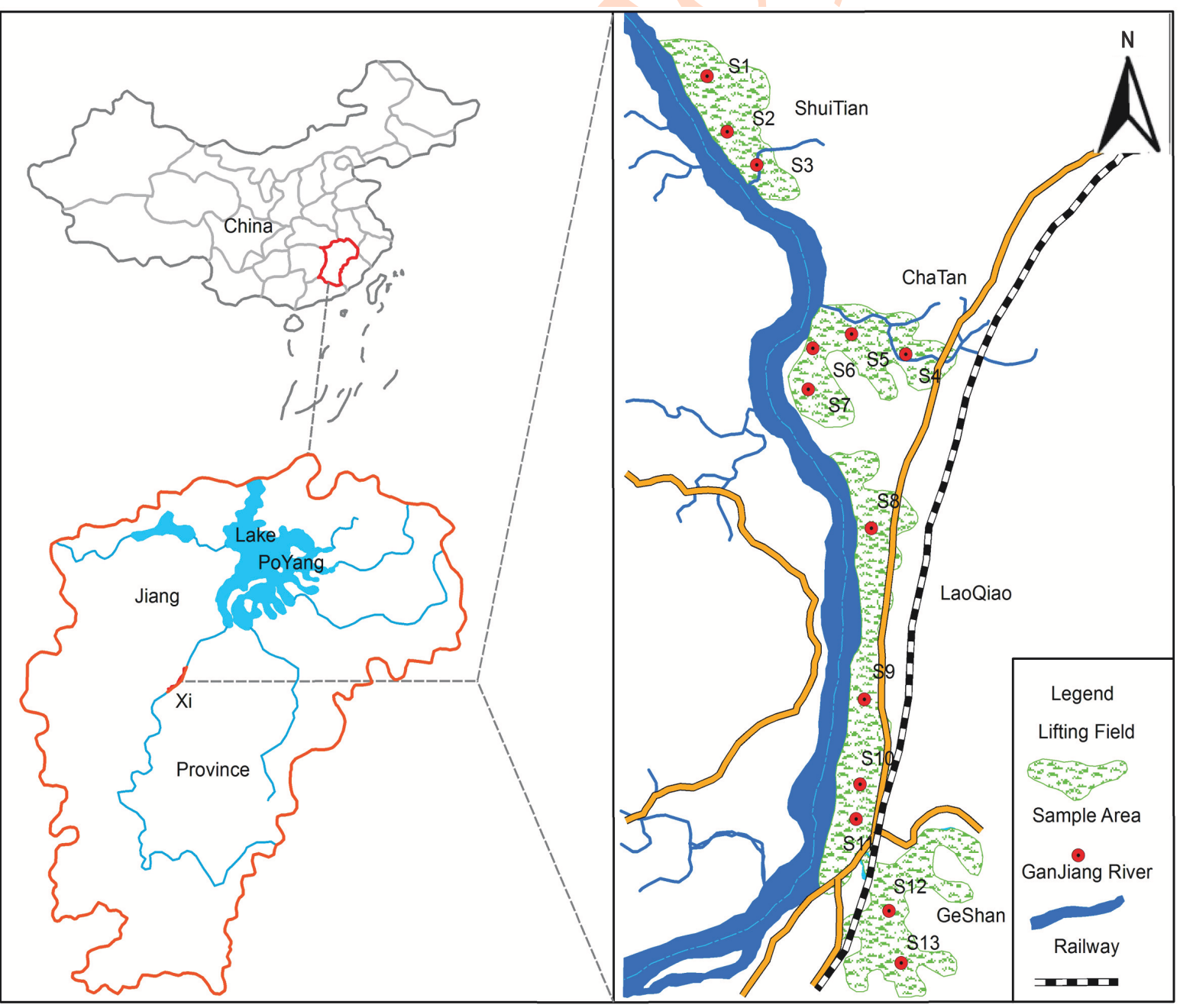

Fig.1 : Map showing the geographical location of sampling sites in Ganjiang region of China 
The function of soil physical and biological properties lies in maintaining soil fertility level and guaranteeing productivity (Mahanta et al., 2014). The results conclude that lifting measures decrease soil quality during the initial stage by reducing vegetation coverage and cultivation layer thickness, weakening the soil aggregate permeability and lowering the soil water holding capacity. Research has indicated that soil environment deterioration and poor plant growth may increase from soil hardening (Hamza et al., 2005). The critical role of long-term fertilization regimes in enhancing soil water moisture and regulating $\mathrm{pH}$ values and soil respiration intensity has been demonstrated (Yang et al., 2011). The current research indicated that the tillage layer soil at the initial stage was inappropriate for microorganisms and nutrient cycles. However, soil physical and biological properties were gradually ameliorated after 1-3 years of cultivation. Similar results were reported by Moffat and Mcneill (1994).

Several studies have indicated that SOC is directly influenced by land management pattern and soil physical properties (Zhang et al., 2012). In red soil, the content and distribution of SOC was significantly related with soil usage. As shown in Fig. 4, SOC contents ranged from 2.4 to $25.3 \mathrm{~g} \mathrm{~kg}^{-1}$, with an average of $13.29 \mathrm{~g} \mathrm{~kg}^{-1}$. The values of SOC in BK ranged from
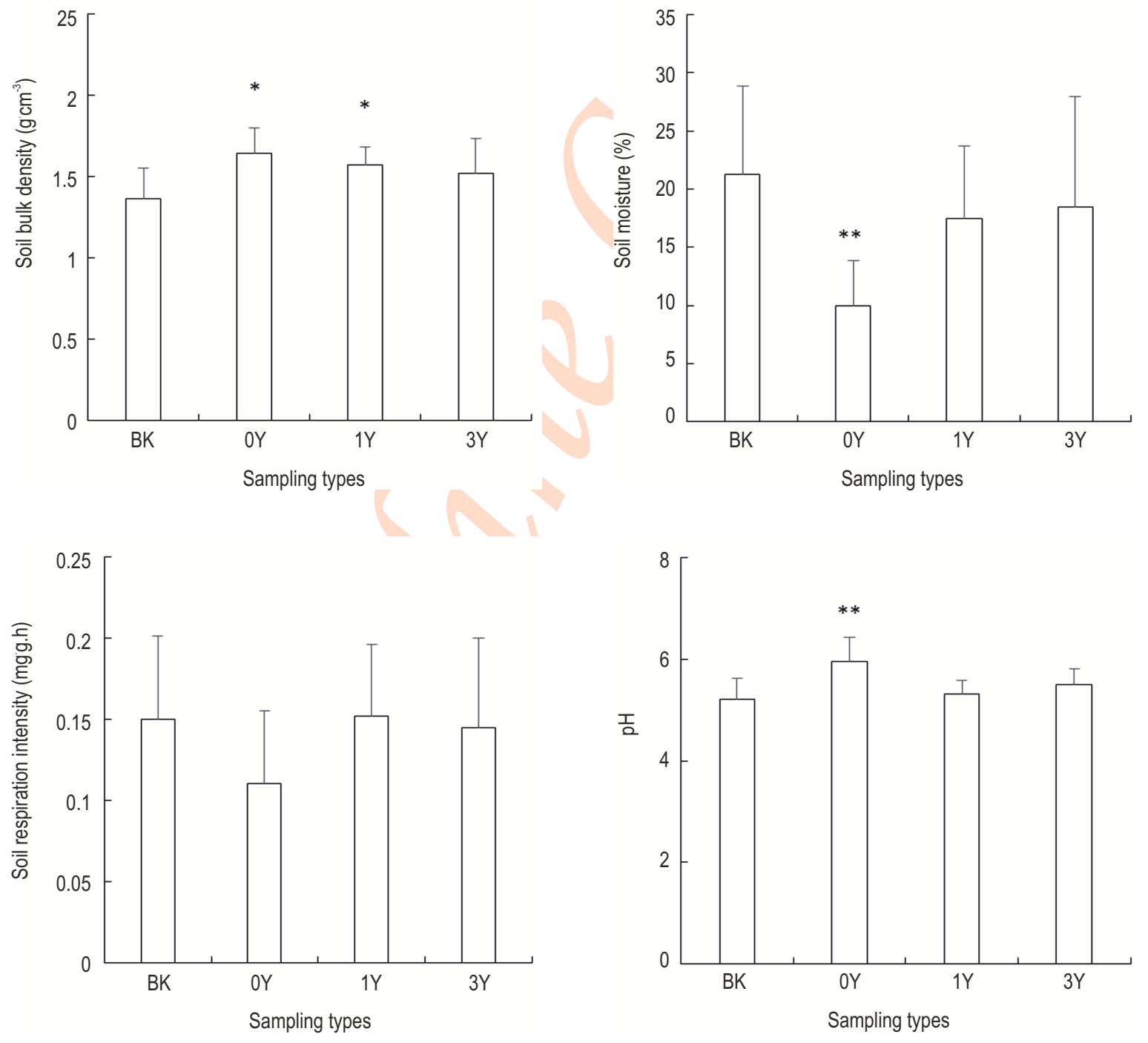

Fig. 2 : The characteristic values of soil density, moisture, respiration intensity and pH in Ganjiang region of China (asterisks indicate $\mathrm{P}<0.05$; double asterisks indicate $\mathrm{P}<0.01$ ) 
9.24 to $25.3 \mathrm{~g} \mathrm{~kg}^{-1}$, with an average of $18.13 \mathrm{~g} \mathrm{~kg}^{-1}$. The minimum average SOC value of $9.03 \mathrm{~g} \mathrm{~kg}^{-1}$ was recorded in OY, which was 0.43 times that of the average BK value. However, the average SOC values in $3 Y$ reached to $16.27 \mathrm{~g} \mathrm{~kg}^{-1}$ after three years cultivating. After calculation, the average $\mathrm{SOC}$ values in the study area were in the following order: $\mathrm{BK}>3 \mathrm{Y}>1 \mathrm{Y}>0 \mathrm{Y}$. Although SOC content decreased significantly after lifting measures, it gradually recovered via scientific management and cultivation.

Total nutrients in soil are the stock of available nutrients, which could reflect the state of soil resource quality (Mitsch and Gosselin, 2000). Previous studies have clarified that total nutrients in paddy soil are one of the important factors effecting rice growth and yield (Li et al., 2011). As shown in Fig. 4(B), 4(C) and 4(D), TN ranged from 0.09 to $1.67 \mathrm{~g} \mathrm{~kg}^{-1}$, with an average of $0.58 \mathrm{~g} \mathrm{~kg}^{-1}$. The lifting measures had a significant effect on TN by disturbing the soil physical characteristics and leading to a sharp decrease. However, TN accumulated gradually and was maintained at a certain level via scientific management and effective fertilization. TP content changes were in a range of $0.99-0.41 \mathrm{~g} \mathrm{~kg}^{-1}$, with an average value of $0.65 \mathrm{~g} \mathrm{~kg}^{-1}$. Similar to TP, TN decreased dramatically after lifting measures and increased gradually to a certain level after a period of cultivation and field management. TK content ranged from 2.4 to $20.7 \mathrm{~g} \mathrm{~kg}^{-1}$, with a mean value of $10.3 \mathrm{~g} \mathrm{~kg}^{-1}$.

Available nutrients can be directly absorbed by plants, which have a close relation with soil fertility level (Li et al., 2011). Available soil nutrients have a close relationship with land use type geomorphic type and soil type (Yang et al., 2010). The maximum value for AN was $83.3 \mathrm{mg} \mathrm{kg}^{-1}$, with an average value of $39.2 \mathrm{mg} \mathrm{kg}^{-1}$. Lifting measures may have destroyed the stability of soil, which led to decrease of AN. The AP content varied between 1.7 and $42.1 \mathrm{mg} \mathrm{kg}^{-1}$, with an average value of $15.12 \mathrm{mg} \mathrm{kg}^{-1}$. Like AN, AP can be seriously affected by lifting measures, but continuously recovered through cultivation. The AK content was in the range of $31.1-132.0 \mathrm{mg} \mathrm{kg}^{-1}$, with an average value of $71.4 \mathrm{mg} \mathrm{kg}^{-1}$. Lifting measures led to a decrease by $44 \%$ for the initial stage but $89 \%$ recovery after three years of farming (Fig. 4E,F and G).

Physical properties influence the accumulation and recycling of soil nutrients (Steven et al., 2005). All soil nutrients studied showed negative correlations with bulk density and positive correlations with soil moisture. A positive correlation was

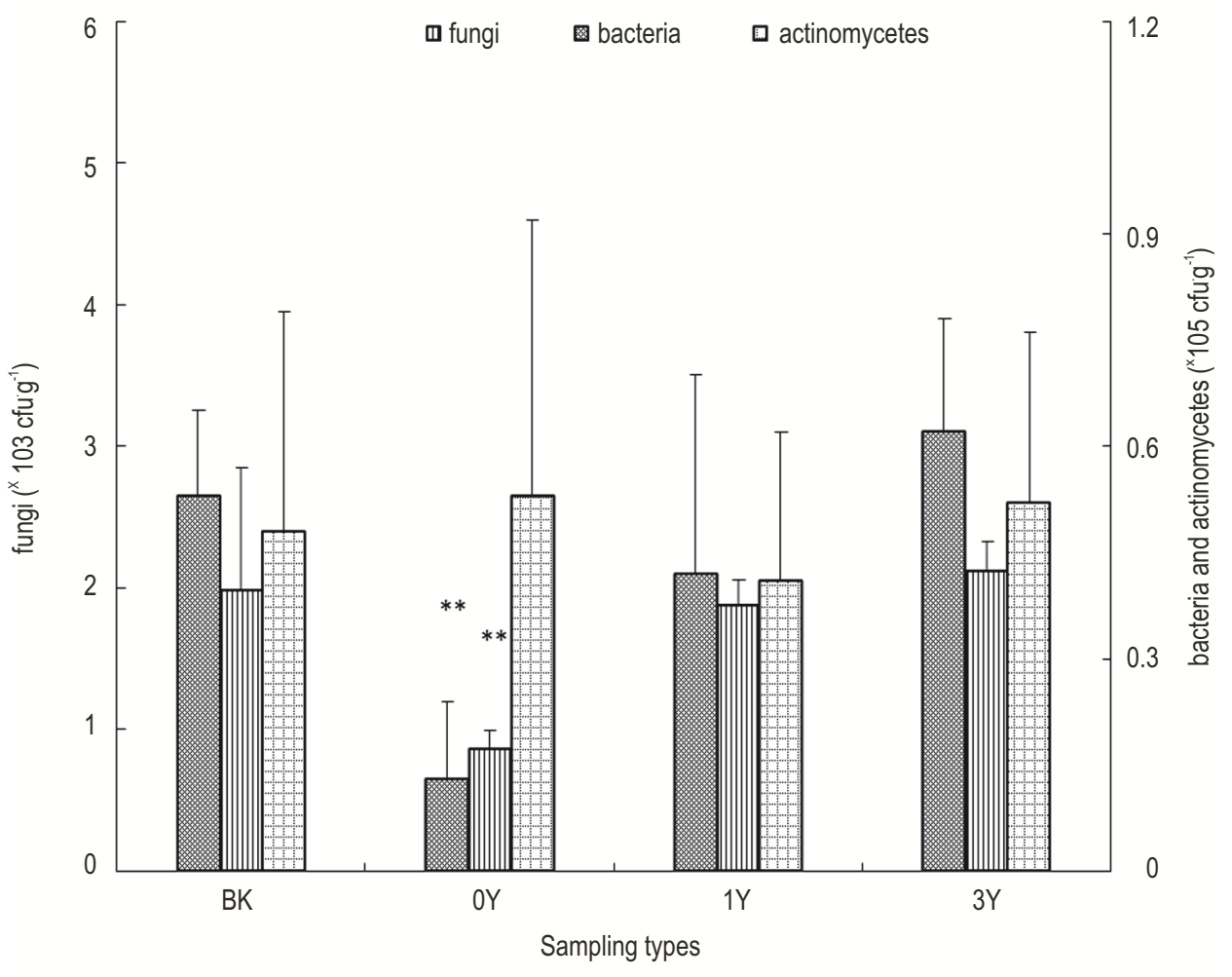

Fig. 3 : Comparison of microbial quantity with BK after different period of lifting measures in Ganjiang region of China (double asterisks indicates $\mathrm{P}<0.01$ ) 

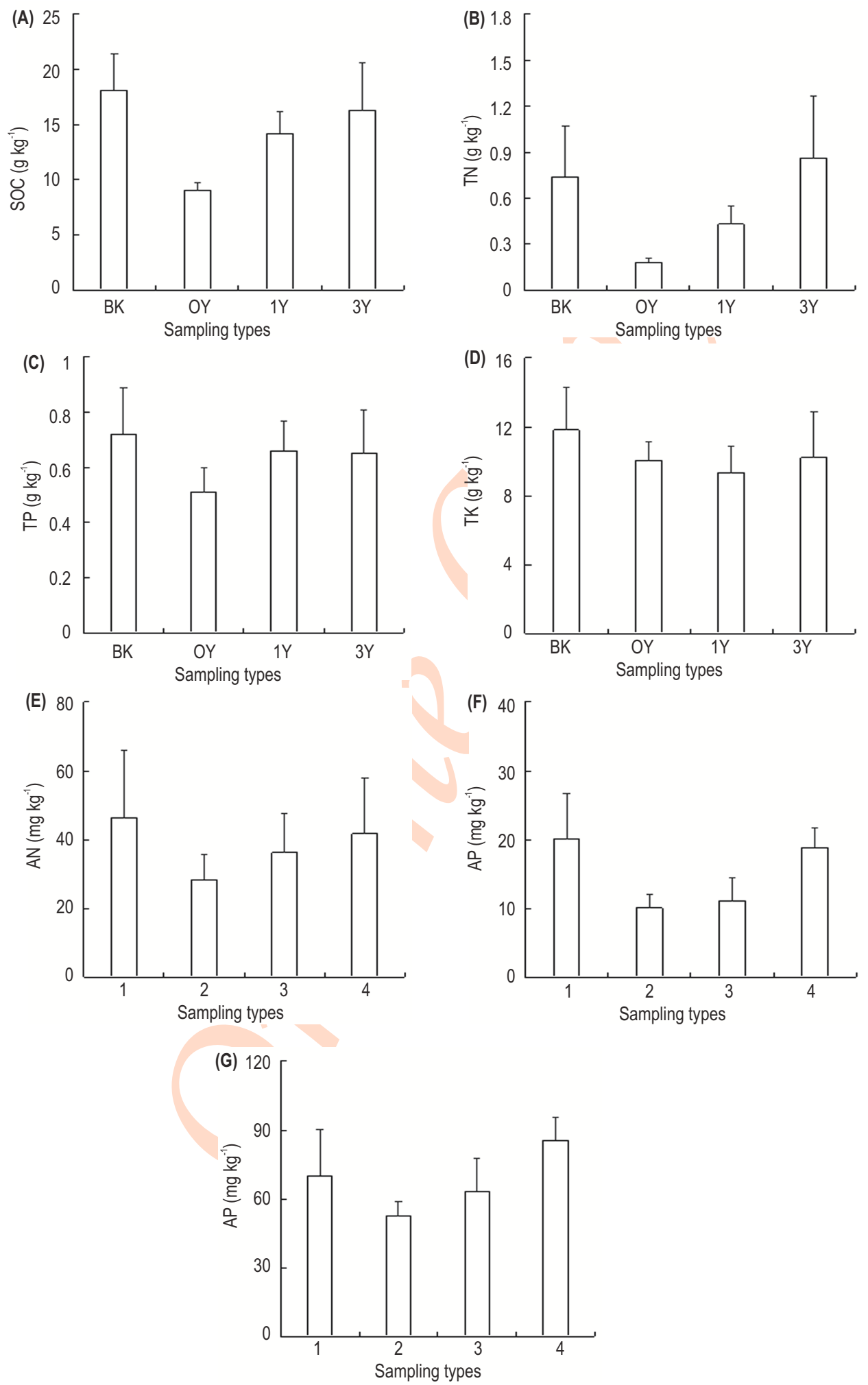

Fig. 4 : Distribution of nutrient and SOC in sediments in Ganjiang region of China (asterisks indicate $P<0.05$; double asterisks indicate $P<0.01$ ) 
also seen among respiration intensity and soil nutrients. There were no significant correlations among soil physical properties and $\mathrm{TN}, \mathrm{TK}$, or AK. It has been well reported that nutrient accumulation in soil is the result of complicated processes effected by multifactorial interactions (Manzoni et al., 2004). SOC and TP contents are easily affected by soil bulk density and moisture, and thus can achieve high levels via sufficient water supply and loose soil. The AN and AK were significantly affected by bulk density and respiration intensity (Stavi et al., 2008).

Microorganisms were also positively correlated with soil physic-chemical characteristics (Parr and Papendick., 1997; Devi and Yadava., 2006). Several studies have shown correlation between microorganisms and soil nutrients (Devi and Yadava., 2006). In the present study, the bacteria, actinomycetes and fungi were positively correlated well with soil nutrients. The quantity of actinomycetes exhibited good positive relationship with SOC, TN, and AN. Similar results were also found for fungi, which correlated well with TN, TK and AN. The amount of bacteria had positive correlations with SOC, TN, TP, AN, and AP. Thus, it was concluded that SOC and nutrients significantly influence soil microbial biomass amounts, and bacteria are more sensitive than fungi and actinomycetes to the effects of SOC and nutrients.

It is well known that soil physical properties and microbial biomass can limit nutrient cycling processes (Compton et al., 2004). In this study, the correlation between nutrients were investigated using correlation analysis. All the nutrient elements correlated well each other. Based on one-way ANOVA, positive correlations existed between SOC and TN (R=0.338), SOC and TP $(R=0.43)$ and $S O C$ and $A N(R=0.894)$. TN was also significantly correlated to SOC, AN, AP and AK. Similar results were found for TP, as it was correlated positively with AN and AP.

Principal component analysis (PCA) is a statistical method that is widely used to convert multiple indicators to fewer meaningful components by discarding some of the nonsignificant information (Xie et al., 2015). In this study, PCA was used to evaluate the degree to which the field lifting affected soil fertility. Four principal components were extracted from the original variables to represent all the information. In general, factor 1 represents all indices except that for TK. Factor 2 explains $20.57 \%$ of the variance with highly positive loading from rapidly AP. The scores of sampling sites added by these four factors were in the following order: $3 Y>B K>1 Y>0 Y$. Owing to effective management, nutrients approached their lowest levels in the early stage after lifting measurement. After that, the soil fertility index increased gradually to 4.53 after three years of management. This indicates that lifting measures cause soil quality to decrease significantly during the early stage and have significant impacts on soil nutrients (Edwards and Jefferies., 2013).

The Integrated Fertility Index (IFI) has been widely used to evaluate soil fertility (Li et al., 2011). In the present study, the correlation coefficients between different variables were used to determine weight (Lv et al., 2004), and the membership values were calculated via the standardized concentration of indices as listed in Li et al. (2011). Furthermore, data of the Second National Soil General Survey was used to determine the function threshold values. The lifting measures resulted in a wide range of IFI values, and the average IFI values of four types of soils were in the following order : $3 \mathrm{Y}>\mathrm{BK}>1 \mathrm{Y}>0 \mathrm{Y}$. Based on the statistical results and standards, 13 sampling sites in this study were divided into four levels: level I (IFI $\geqq 0.75$ ), level II (IFI: 0.75-0.5), level III (IFI: $0.5-0.25)$, and level IV (IFI $\leqq 0.25)$. The IFI values of BK and $3 Y$ were mainly focused in level II and III, with an average IFI value of 0.44 for BK and 0.47 for $3 Y$. The IFI values of $O Y$ and $1 Y$ were lower than level IV, indicating that $\mathrm{OY}$ and $1 \mathrm{Y}$ were heavily affected by the lifting measures.

This study revealed that lifting measures can cause a significant quality decrease of paddy soil planted along the Gan River in Jiangxi province, which was mainly characterized by significant changes in physical and biological soil properties. Permeability and holding capacity of soil were largely destroyed during the early stage and were unsuitable for microorganisms and nutrient cycles. Nevertheless, soil physical and biological properties gradually ameliorated after several years' scientific management and cultivation. Hence, no significant difference was seen in nutrient levels between the original planting areas and areas after three years of cultivation.

\section{Acknowledgments}

This study was supported by National Natural Science Foundation of China (51469017; 51579127; 51679141), Ministry of Water Resources (201401039), Project of Jiangxi Provincial Technology Department (20142BBF60012; 20142BAB213024), Open Foundation of JiangXi Engineering Research Center of Water Engineering Safety and Resources Efficient Utilization ( OF201608).

\section{References}

Castilho, D.P., S. Cristina, C. Miguel, D. Znahí and B. Jose Camilo: Effect of land use changes in Eastern Amazonia on soil chemical, physical, and biological attributes. Soil Sci., 181, 133-147 (2016).

Compton, J.E., L.S. Watrud, L.A.Porteous and S. DeGrood: Response of soil microbial biomass and community composition to chronic nitrogen additions at Harvard forest. Forest Ecol. Manag., 196, 143-158 (2004).

Devi, N.B. and P.S. Yadava: Seasonal dynamics in soil microbial C, N and $\mathrm{P}$ in a mixed-oak forest ecosystem of Manipur, North-east India. Appl. Soil Ecol., 31, 220-227(2006).

Edwards, K.A. and R.J. Jefferies: Inter-annual and seasonal dynamics of soil microbial biomass and nutrientsin wet and dry low-Arctic sedge meadows. Soil Biol. Biochem., 57, 83-90 (2013).

Helfricha, M., B. Ludwig, C. Thomsc, G. Gleixnerd and H. Flessa: The role of soil fungi and bacteria in plant litter decomposition and macroaggregate formation determined using phospholipid fatty acids. Appl. Soil Ecol., 96, 261-264 (2015). 
Hobara, S., T. Osono, D. Hirose, K. Noro, M. Hirot and R. Benner: The roles of microorganisms in litter decomposition and soil formation. Biogeochemistry, 118, 471-486 (2014).

Hou,X.Q., R. Li, Z.F. Jia, Q.F. Han, B.P. Yang and J.F. Nie: Effects of rotational tillage practices on soil structure, organic carbon concentration and crop yields in semi-arid areas of northwest China. Soil Use Manage., 28, 551-558 (2012).

Hamza, M.A. and W.K. Anderson : Soil compaction in cropping systems: A review of the nature, causes and possible solutions. Soil Till. Res., 82, 121-145(2005).

Li, Z.W., J.Q. Huang, Y.Y. Li, W. Guo and J.F. Zhu: Assessment on soil fertility of Dongting Lake wetland area (China) based on GIS and fuzzy evaluation. Cent. South Univ. Technol, 18, 1465-1472 (2011).

Lozano, L.A., C.G. Soracco, R. Villarreal, J.M. Ressia, G.O. Sarli and R.R. Filgueira: Soil physical quality and soybean yield as affected by chiseling and cubsoiling of a no-till soil. Rev. bras. ciênc. Solo., 40, 1-12(2016)

Lv, X., J.M. Kou and H.W. Li: Fuzzy integrative evaluation of soil fertility based on GIS. Agricul. Res. Arid Areas, 22, 56-59 (2004).

Mahanta, K., D.K. Jha, D.J. Rajkhowa and M. Kumar: Isolation and evaluation of native cellulose degrading microorganisms for efficient bioconversion of weed biomass and rice straw. J. Environ. Biol., 35, 721(2014).

Mandal, S., B.C. Verma, G.I. Ramkrushna,R.K. Singh and D.J. Rajkhowa: Characterization of biochar obtained from weeds and its effect on soil properties of north eastern region of india. J. Environ. Biol., 36, 499-505 (2015).

Manzoni, S., A. Porporato, P. D' Odorico, F. Laio and I. Rodrigueziturbe: Soil nutrient cycles as a nonlinear dynamical system. Nonlinear Pro. Geoph., 11, 589-598 (2004).

Masciandaro, G., C. Macci, E. Peruzzi, B. Ceccanti and S. Doni: Organic matter-microorganism-plant in soil bioremediation: A synergic approach. Rev. Environ. Sci. Biotechnol., 12, 399-419 (2013).

Moffat, A.J. and J.D. Mcneill: Reclaiming disturbed land for forestry [M]. London: HMSO, p. 112(1994).

Mitsch, W.J. and K.J.G. Gosselin : Wetlands. New York : John Wiley \& Sons, pp. 155-204 (2000).

Masoom, H., D. Courtiermurias, H. Farooq, R. Soong and B.P. Kelleher : Soil organic matter in its native state: Unravelling the most complex biomaterial on earth. Sci. Technol., 50, 1670-1680 (2016).
Mlih, R., B.O.L. Roland, A. Wulf and B. Nadhem: Soil organic matter amendments in date palm groves of the Middle Eastern and North African region: A mini-review. Arid Land, 8, 77-92 (2016).

Parr, J.F. and R.I. Papendick: Soil quality, relationship and strategies for sustainable dryland farming system. Ann. Arid Zones, 36, 181-191 (1997).

Sun, B., D. Paul, S.C. Hallett, J.D. Tim and W.H. David: Distribution of soil carbon and microbial biomass in arable soils under different tillage regimes. Plant Soil, 338,17-25 (2011).

Sutton,R. and G. Sposito : Molecular structure in soil humic substances: The new view. Environ. Sci. Technol., 39, 9009-15 (2006).

Smebye, A., V. Alling, R.D. Vogt, T.C. Gadmar, J. Mulder, G. Cornelissen and S.E. Hale : Biochar amendment to soil changes dissolved organic matter content and composition. Chemosphere, 142, 100$105(2015)$.

Stavi, Ilan., D.U. Eugene, H. Lavee and P. Sarah: Grazing-induced spatial variability of soil bulk density and content of moisture organic carbon and calcium carbonate in a semi-arid range land. Catena, 75, 288-296 (2008).

Steven, V. and M.A. Cavigelli : Soil physical properties and aggregateassociated $\mathrm{C}, \mathrm{N}$ and $\mathrm{P}$ distributions in organic and conventional cropping systems. Soil Sci., 170, 822-831 (2005).

Tang, Z.X. and Z.R. Wang: Primary analysis of the flood control dispatch of Xiajiang Reservoir. Jiangxi Hydraulic Sci. Technol., 29, 233-236 (2003).

Xie, L.W., J. Zhong, F.F. Chen, F.X. Cao, J.J. Li and L.C. Wu: Evaluation of soil fertility in the succession of karst rocky desertification using principal component analysis. Solid Earth, 6, 3333-3359 (2015).

Yang, X., P. Li, S. Zhang, B. Sun and X. Chen: Long-term-fertilization effects on soil organic carbon, physical properties, and wheat yield of a loess soil. J. Plant Nutr. Soil Sci.,174, 775-784 (2011).

Yang, H., L.K. Zhang, J.H. Cao and Y.L. Hou : Effects of different land use types on soil organic carbon and carbon management index in karstarea. Agricul. Sci. Technol., 11, 136-139 (2010)

Zhang, W., M. Xu, X. Wang, Q. Huang, J.Nie and Z. Li: Effects of organic amendments on soil carbon sequestration in paddy fields of subtropical China. J. Soil. Sediment., 12, 457-470 (2012).

Zhen, Y.J: Analysis of the significance and process of the construction of the lifting measures in Water Control Projects. Jiangxi Building Materials, 157, 117-118(2015). 